\title{
Existence results for an impulsive Sturm-Liouville boundary value problems with mixed double parameters
}

\section{Yulin Zhao, Li Huang* and Qiming Zhang}

"Correspondence: lihung09@sina.cn School of Science, Hunan University of Technology, Zhuzhou, Hunan 412007, P.R. China

\begin{abstract}
In this paper, a class of fourth-order impulsive differential equations depending on two control parameters is investigated. The existence and multiplicity of solutions are obtained by means of the variational methods and the critical point theory. Finally, an example which supports our theoretical results is also presented.
\end{abstract}

Keywords: impulsive effects; Sturm-Liouville boundary value problem; variational methods; fourth-order

\section{Introduction}

In this paper we will investigate the existence of infinitely many solutions for the following Sturm-Liouville boundary value problem:

$$
\begin{gathered}
u^{(i v)}(t)-u^{\prime \prime}(t)+u(t)=\lambda f(t, u(t))+\mu g(t, u(t)), \quad t \neq t_{j}, \text { a.e. } t \in[0, T], \\
(B C):\left\{\begin{array}{l}
\alpha_{1} u(0)-\beta_{1} u^{\prime}(0)=0, \quad \alpha_{2} u(T)+\beta_{2} u^{\prime}(T)=0, \\
\alpha_{1} u^{\prime \prime}(0)-\beta_{1} u^{\prime \prime \prime}(0)=0, \quad \alpha_{2} u^{\prime \prime}(T)+\beta_{2} u^{\prime \prime \prime}(T)=0,
\end{array}\right.
\end{gathered}
$$

with impulsive conditions

$$
\triangle u^{\prime \prime}\left(t_{j}\right)=I_{1 j}\left(u^{\prime}\left(t_{j}\right)\right), \quad-\triangle u^{\prime \prime \prime}\left(t_{j}\right)=I_{2 j}\left(u\left(t_{j}\right)\right), \quad j=1,2, \ldots, l,
$$

where $\lambda$ and $\mu$ are two real parameters; $\alpha_{1}, \alpha_{2}, \beta_{1}, \beta_{2}$ are positive constants, $f, g$ : $[0, T] \times \mathbf{R} \rightarrow \mathbf{R}$ are $L^{2}$-Carathéodory functions, $I_{1 j}, I_{2 j}: \mathbf{R} \rightarrow \mathbf{R}$ are continuous functions, $j=1,2, \ldots, l, 0=t_{0}<t_{1}<t_{2}<\cdots<t_{l}<t_{l+1}=T, \Delta u^{\prime \prime \prime}\left(t_{j}\right)=u^{\prime \prime \prime}\left(t_{j}^{+}\right)-u^{\prime \prime \prime}\left(t_{j}^{-}\right), \Delta u^{\prime \prime}\left(t_{j}\right)=$ $u^{\prime \prime}\left(t_{j}^{+}\right)-u^{\prime \prime}\left(t_{j}^{-}\right)$, where $y\left(t_{j}^{+}\right), y\left(t_{j}^{-}\right)$denote the right and left limits of $y(t)$ at $t=t_{j}$, respectively. We refer to the impulsive problems (1.1)-(1.3) as (IP).

In recent years, the existence and multiplicity of solutions for Sturm-Liouville boundary value problems (for short BVPs) have been studied extensively in the literature (see, for example, [1-10] and references therein). Some classical tools have been used to deal with such problems in the literature, such as the method of upper and lower solutions with the monotone iterative technique, the coincidence degree theory of Mawhin, and some fixed point theorems in cones (see [1-7]). Recently, many researchers [11-16] have studied the 
existence and multiplicity of solutions of impulsive problems by using variational methods and critical point theory. Moreover, among the papers where impulsive differential equations are investigated by using variational methods, most are for a second-order differential equation, whereas the ones for a fourth-order are mostly about Dirichlet boundary conditions and Neumann boundary conditions. In [13], the authors obtained sufficient conditions that guarantee the existence of at least one solution or infinitely many classical solutions of the following equations:

$$
\left\{\begin{array}{l}
u^{(i v)}(t)+A u^{\prime \prime}(t)+B u(t)=f(t, u(t)), \quad t \neq t_{j}, \text { a.e. } t \in[0, T] \\
-\triangle u^{\prime \prime}\left(t_{j}\right)=I_{1 j}\left(u^{\prime}\left(t_{j}\right)\right), \quad j=1,2, \ldots, l, \\
-\triangle u^{\prime \prime \prime}\left(t_{j}\right)=I_{2 j}\left(u\left(t_{j}\right)\right), \quad j=1,2, \ldots, l, \\
u(0)=u(T)=u^{\prime \prime}\left(0^{+}\right)=u^{\prime \prime}\left(T^{-}\right)=0 .
\end{array}\right.
$$

Cabada and Tersian in [17] considered the existence and the multiplicity of solutions for the following fourth-order differential equations:

$$
\left\{\begin{array}{l}
u^{(i v)}(t)-a u^{\prime \prime}(t)+b(t) u(t)=c(t)|u|^{p-2} u, \quad t \in[0, T], t \neq t_{j} \\
\triangle u^{\prime \prime \prime}\left(t_{j}\right)=I_{1 j}\left(u^{\prime}\left(t_{j}\right)\right), \quad \Delta u^{\prime \prime}\left(t_{j}\right)=-I_{2 j}\left(u\left(t_{j}\right)\right), \quad j=1,2, \ldots, l \\
u(0)=u(T)=u^{\prime \prime}(0)=u^{\prime \prime}(T)=0
\end{array}\right.
$$

By using the minimization, the mountain-pass and Clarke theorems, they obtained the existence of at least one or infinitely many nonzero solutions.

However, to our knowledge, besides [8, 18-20] for second-order impulsive differential equations subject to Sturm-Liouville boundary conditions, the study of solutions for a fourth-order Sturm-Liouville boundary value problem with impulsive effects using variational methods has received considerably less attention (see, for example, [21-23]). In [19], Tian and Ge studied the following Sturm-Liouville boundary value problems with impulsive effects:

$$
\left\{\begin{array}{l}
-\left(\rho(t) \varphi_{p}\left(u^{\prime}(t)\right)\right)^{\prime}+s(t) \varphi_{p}(u(t))=f(t, u(t)), \quad t \neq t_{j}, \text { a.e. } t \in[a, b] \\
-\triangle \varphi_{p}\left(\rho(t) u^{\prime}\left(t_{j}\right)\right)=I_{j}\left(u\left(t_{j}\right)\right), \quad j=1,2, \ldots, l, \\
\alpha_{1} u(a)-\beta_{1} u^{\prime}(a)=A, \quad \alpha_{2} u(b)+\beta_{2} u^{\prime}(b)=B .
\end{array}\right.
$$

They essentially proved that when $f$ and $I_{j}$ satisfy some conditions, the problem (1.4) has at least two positive solutions via variational methods.

By means of the mountain-pass lemma and the linking theorem, Tian and Liu [23] studied the Sturm-Liouville boundary value problems:

$$
u^{(i v)}(t)-u^{\prime \prime}(t)+u(t)=f(t, u(t)), \quad t \neq t_{j} \text {, a.e. } t \in[0, T],
$$

with boundary conditions (1.2) and impulsive conditions

$$
-\triangle u^{\prime \prime}\left(t_{j}\right)=I_{1 j}\left(u^{\prime}\left(t_{j}\right)\right), \quad-\triangle u^{\prime \prime \prime}\left(t_{j}\right)=I_{2 j}\left(u\left(t_{j}\right)\right), \quad j=1,2, \ldots, l,
$$

where $f \in C([0, T] \times \mathbf{R}, \mathbf{R})$. The main result is as follows.

Theorem A ([23], Theorem 3.1) Suppose that the following conditions hold. 
(C1) There exist constants $\mu>2$ and $r \geq 0$ such that for $|\xi| \geq r$,

$$
0<\mu F(t, \xi) \leq \xi f(t, \xi)
$$

(C2) The impulsive functions $I_{2 j}$ satisfy sublinear growth, that is, there exist constants $a_{j}>0, b_{j}>0$, and $\gamma_{j} \in[0,1), j=1,2, \ldots$, l such that $\left|I_{2 j}(u)\right| \leq a_{j}+b_{j}|u|^{\gamma_{j}}$.

(C3) The impulsive functions $I_{1 j}, j=1,2, \ldots$, l are bounded.

(C4) $f(t, u)=o(|u|), I_{1 j}(u)=o(|u|), I_{2 j}(u)=o(|u|)$ as $|u| \rightarrow 0, j=1,2, \ldots, l$.

Then the problem (1.5), (1.6), and (1.2) has at least one nontrivial solution.

Based on the knowledge mentioned above, the purpose of the present paper is to establish some new criteria for (IP) to have infinitely many weak solutions via variational methods and critical point theory. We will prove the existence of an interval $\Lambda \subset(0,+\infty)$ with the following property: for each $\lambda \in \Lambda$ there exists $\mu^{*}>0$ such that, for each $\mu \in\left[0, \mu^{*}\right)$, the problem (IP) admits infinitely many solutions when the primitive $F$ of $f$ satisfies a suitable oscillatory behavior either at infinity or at the origin, and the primitive $G$ of $g$ exhibits appropriate growth (see Theorems 3.1 and 3.6).

\section{Preliminaries}

Hereafter, let $X:=\left\{u \in H^{2}(0, T) \mid \alpha_{1} u(0)-\beta_{1} u^{\prime}(0)=0, \alpha_{2} u(T)+\beta_{2} u^{\prime}(T)=0\right\}$ be endowed with the inner product

$$
(u, v)=\int_{0}^{T} u^{\prime \prime}(t) v^{\prime \prime}(t)+u^{\prime}(t) v^{\prime}(t)+u(t) v(t) d t, \quad \forall u, v \in X
$$

which induces the norm

$$
\|u\|_{X}=\left(\int_{0}^{T}\left|u^{\prime \prime}(t)\right|^{2}+\left|u^{\prime}(t)\right|^{2}+|u(t)|^{2} d t\right)^{1 / 2}
$$

Since $X$ is a closed subspace of $H^{2}(0, T),\left(X ;\|u\|_{X}\right)$ is a Banach space. Define the usual norms of $C^{1}([0, T]), L^{2}(0, T)$, respectively, as

$$
\|u\|_{0}=\max \left\{\max _{t \in[0, T]}|u(t)|, \max _{t \in[0, T]}\left|u^{\prime}(t)\right|\right\}, \quad\|u\|_{L^{2}}=\left(\int_{0}^{T}|u(t)|^{2} d t\right)^{1 / 2}
$$

Lemma 2.1 ([23], Lemma 2.5) Let $u \in X$. Then $\|u\|_{0} \leq M\|u\|_{X}$, where

$$
M=\frac{1}{\sqrt{T}}+\sqrt{T}
$$

According to Lemma 2.1, we have

$$
\|u\|_{L^{2}}=\left(\int_{0}^{T}|u(t)|^{2} d t\right)^{1 / 2} \leq\left(\int_{0}^{T} M^{2}\|u\|_{X}^{2} d t\right)^{1 / 2}=(1+T)\|u\|_{X} .
$$

Throughout the sequel, let $f, g:[0, T] \times \mathbf{R} \rightarrow \mathbf{R}$ be two $L^{2}$-Carathéodory functions. Recall that a function $\phi:[0, T] \times \mathbf{R} \rightarrow \mathbf{R}$ is said to be an $L^{2}$-Carathéodory function, if

(A1) $t \rightarrow \phi(t, u)$ is measurable for every $u \in \mathbf{R}$; 
(A2) $u \rightarrow \phi(t, u)$ is continuous for almost every $t \in[0, T]$;

(A3) for each $\rho>0$, the function $l_{\rho}(t):=\sup _{|u| \leq \rho}|\phi(t, u)|$ belongs to $L^{2}(0, T)$.

In order to study (IP), we consider the functionals $\Phi, \Psi: X \rightarrow \mathbf{R}$ defined by

$$
\begin{aligned}
\Phi(u):= & \frac{1}{2}\|u\|_{X}^{2}+\sum_{j=1}^{l} \int_{0}^{u^{\prime}\left(t_{j}\right)} I_{1 j}(t) d t+\sum_{j=1}^{l} \int_{0}^{u\left(t_{j}\right)} I_{2 j}(t) d t \\
& +\frac{\alpha_{1}}{2 \beta_{1}}(u(0))^{2}+\frac{\alpha_{2}}{2 \beta_{2}}(u(T))^{2}, \\
\Psi(u):= & \int_{0}^{T} F(t, u(t)) d t+\frac{\mu}{\lambda} \int_{0}^{T} G(t, u(t)) d t,
\end{aligned}
$$

where $F(t, u)=\int_{0}^{u} f(t, x) d x, G(t, u)=\int_{0}^{u} g(t, x) d x$.

It follows from the properties of $f, g$ and the continuity of $I_{1 j}, I_{2 j}, j=1,2, \ldots, l$, that $\Phi, \Psi$ are Gâteaux differentiable functionals whose Gâteaux derivatives are given by

$$
\begin{aligned}
\Phi^{\prime}(u)(v)= & \int_{0}^{T}\left(u^{\prime \prime}(t) v^{\prime \prime}(t)+u^{\prime}(t) v^{\prime}(t)+u(t) v(t)\right) d t+\sum_{j=1}^{l} I_{1 j}\left(u^{\prime}\left(t_{j}\right)\right) v^{\prime}\left(t_{j}\right) \\
& +\sum_{j=1}^{l} I_{2 j}\left(u\left(t_{j}\right)\right) v\left(t_{j}\right)+\frac{\alpha_{1}}{\beta_{1}} u(0) v(0)+\frac{\alpha_{2}}{\beta_{2}} u(T) v(T)
\end{aligned}
$$

and

$$
\Psi^{\prime}(u)(v)=\int_{0}^{T} f(t, u(t)) v(t) d t+\frac{\mu}{\lambda} \int_{0}^{T} g(t, u(t)) v(t) d t
$$

for every $v \in X$.

By a classical solution of (IP), we mean a function

$$
u \in\left\{z \in C([0, T]):\left.z\right|_{\left[t_{j}, t_{j+1}\right]} \in H^{4}\left(\left[t_{j}, t_{j+1}\right]\right), j=0,1,2, \ldots, l-1 ;\left.z\right|_{\left[t_{l}, T\right]} \in C^{3}\left(\left[t_{l}, T\right]\right)\right\}
$$

that satisfies (1.1) a.e. on $[0, T]$, the boundary conditions (1.2) and the impulsive condition (1.3). If $f, g$ are continuous on $[0, T] \times \mathbf{R}$, therefore each classical solution $u \in C^{3}\left(\left[t_{j}, t_{j+1}\right]\right)$, $j=1, \ldots, l$, and satisfies (1.1) for all $t \in[0, T] \backslash\left\{t_{1}, \ldots, t_{l}\right\}$.

Definition 2.2 A function $u \in X$ is said to be a weak solution of (IP) if $u$ satisfies

$$
\begin{aligned}
0= & \int_{0}^{T}\left(u^{\prime \prime}(t) v^{\prime \prime}(t)+u^{\prime}(t) v^{\prime}(t)+u(t) v(t)\right) d t+\sum_{j=1}^{l} I_{1 j}\left(u^{\prime}\left(t_{j}\right)\right) v^{\prime}\left(t_{j}\right)+\sum_{j=1}^{l} I_{2 j}\left(u\left(t_{j}\right)\right) v\left(t_{j}\right) \\
& -\lambda \int_{0}^{T} f(t, u(t)) v(t) d t-\mu \int_{0}^{T} g(t, u(t)) v(t) d t+\frac{\alpha_{1}}{\beta_{1}} u(0) v(0)+\frac{\alpha_{2}}{\beta_{2}} u(T) v(T),
\end{aligned}
$$

for any $v \in X$.

Arguing as in Lemma 2.4 of [23], one obtains the following result.

Lemma 2.3 The function $u \in X$ is a weak solution of $(I P)$ if and only if $u$ is a classical solution of $(I P)$. 
Put

$$
E_{\lambda, \mu}:=\Phi(u)-\lambda \Psi(u), \quad \forall u \in X
$$

By standard arguments, one infers that the critical points of the functional $E_{\lambda, \mu}$ are the weak solutions of (IP). Hence, in view of Lemma 2.3, the critical points of (IP) are the classical solutions.

Our analysis is mainly based on the following critical points theorem of Bonanno and Molica Bisci [24], which is a more precise version of Ricceri's variational principle [25], Theorem 2.5:

Lemma 2.4 ([24], Theorem 2.1) Let $X$ be a reflexive real Banach space. Let $\Phi, \Psi: X \rightarrow \mathbf{R}$ be two Gâteaux differentiable functionals such that $\Phi$ is sequentially weakly lower semicontinuous, strongly continuous, and coercive and $\Psi$ is sequentially weakly upper semicontinuous. For every $r>\inf _{X} \Phi$, put

$$
\varphi(r):=\inf _{u \in \Phi^{-1}(-\infty, r)} \frac{\sup _{v \in \Phi^{-1}(-\infty, r)} \Psi(v)-\Psi(u)}{r-\Phi(u)}
$$

and

$$
\gamma:=\lim \inf _{r \rightarrow+\infty} \varphi(r), \quad \delta:=\lim \inf _{r \rightarrow\left(\inf _{X} \Phi\right)^{+}} \varphi(r) .
$$

Then

(a) For every $r>\inf _{X} \Phi$ and every $\lambda \in\left(0, \frac{1}{\varphi(r)}\right)$ the restriction of the functional $\Phi-\lambda \Psi$ to $\Phi^{-1}(-\infty, r)$ admits a global minimum, which is a critical point (local minimum) of $\Phi-\lambda \Psi$ in $X$.

(b) If $\gamma<+\infty$ then, for each $\lambda \in\left(0, \frac{1}{\gamma}\right)$, the following alternative holds: either the functional $\Phi-\lambda \Psi$ has a global minimum, or there exists a sequence $\left\{u_{n}\right\}$ of critical points (local minima) of $\Phi-\lambda \Psi$ such that $\lim _{n \rightarrow+\infty} \Phi\left(u_{n}\right)=+\infty$.

(c) If $\delta<+\infty$ then, for each $\lambda \in\left(0, \frac{1}{\delta}\right)$, the following alternative holds: either there exists a global minimum of $\Phi$ which is a local minimum of $\Phi-\lambda \Psi$, or there exists a sequence $\left\{u_{n}\right\}$ of pairwise distinct critical points (local minimum) of $\Phi-\lambda \Psi$, with $\lim _{n \rightarrow+\infty} \Phi\left(u_{n}\right)=\inf _{X} \Phi$, which weakly converges to a global minimum of $\Phi$.

\section{Main results and proof}

In this section, we will state and prove our main results.

For convenience, put

$$
\begin{aligned}
& \Gamma(T):=\frac{\alpha_{1} \beta_{2}+\alpha_{2} \beta_{1}}{6 \alpha_{1} \alpha_{2}}+\frac{6 \alpha_{1}^{3} T-8 \beta_{1}^{3}}{3 \alpha_{1}\left(4 \beta_{1}+\alpha_{1} T\right)^{2}}+\frac{6 \alpha_{2}^{3} T-8 \beta_{2}^{3}}{3 \alpha_{2}\left(4 \beta_{2}+\alpha_{2} T\right)^{2}}+\frac{T}{3}, \\
& \hbar(T, M):=\Gamma(T)+\frac{8 \alpha_{1} \beta_{1}}{\left(4 \beta_{1}+\alpha_{1} T\right)^{2}}+\frac{8 \alpha_{2} \beta_{2}}{\left(4 \beta_{2}+\alpha_{2} T\right)^{2}}+\frac{1}{2}\left(k_{1}+k_{2}\right) M^{2}, \\
& M_{*}:=1-\left(k_{1}+k_{2}\right) M^{2}, \\
& \lambda_{1}:= \begin{cases}\frac{\hbar(T, M)}{\limsup _{\xi \rightarrow+\infty} \frac{\int_{T / 4}^{3 T / 4} F(t, \xi) d t}{\xi^{2}},} & \text { if } \lim \sup _{\xi \rightarrow+\infty} \frac{\int_{T / 4}^{3 T / 4} F(t, \xi) d t}{\xi^{2}}<+\infty, \\
0, & \text { if } \limsup _{\xi \rightarrow+\infty} \frac{\int_{T / 4}^{3 T / 4} F(t, \xi) d t}{\xi^{2}}=+\infty,\end{cases}
\end{aligned}
$$




$$
\lambda_{2}:=\frac{M_{*}}{2 M^{2} \cdot \liminf _{\xi \rightarrow+\infty} \frac{\int_{0}^{T} \max |x| \leq \xi}{\xi^{2}} F(t, x) d t} .
$$

Throughout this paper, we assume that

(H0) there exist two positive constants $k_{1}$ and $k_{2}$ such that

$$
\left|\int_{0}^{\eta} I_{i j}(s) d s\right| \leq \frac{k_{i}}{2 l} \eta^{2}, \quad \text { for all } \eta \in \mathbf{R}, j=1, \ldots, l \text { and } i=1,2 \text {. }
$$

Theorem 3.1 Let $f:[0, T] \times \mathbf{R} \rightarrow \mathbf{R}$ be an $L^{2}$-Carathéodory function. Furthermore, assume that

(H1) $\left\{t_{1}, t_{2}, \ldots, t_{l}\right\} \subseteq\left[\frac{T}{4}, \frac{3 T}{4}\right]$ and $\left(k_{1}+k_{2}\right) M^{2}<1$;

(H2) $F(t, u) \geq 0$ for all $(t, u) \in\left(\left[0, \frac{T}{4}\right] \cup\left[\frac{3 T}{4}, T\right]\right) \times \mathbf{R}$;

(H3)

$$
\liminf _{\xi \rightarrow+\infty} \frac{\int_{0}^{T} \max _{|u| \leq \xi} F(t, u) d t}{\xi^{2}}<\frac{M_{*}}{2 M^{2} \hbar(T, M)} \cdot \limsup _{\xi \rightarrow+\infty} \frac{\int_{T / 4}^{3 T / 4} F(t, \xi) d t}{\xi^{2}} .
$$

Then $\lambda_{1}<\lambda_{2}$ and for every arbitrary $\lambda \in \Lambda:=\left(\lambda_{1}, \lambda_{2}\right)$, and for every arbitrary $L^{2}$ Carathéodory function $g:[0, T] \times \mathbf{R} \rightarrow \mathbf{R}$ such that

$$
\begin{aligned}
& G(t, u) \geq 0 \quad \text { for all }(t, u) \in[0, T] \times \mathbf{R}, \\
& G_{\infty}:=\limsup _{\xi \rightarrow+\infty} \frac{\int_{0}^{T} \max _{|u| \leq \xi} G(t, u) d t}{\xi^{2}}<+\infty,
\end{aligned}
$$

and for every $\mu \in \Theta:=\left[0, \mu_{*}\right)$, where

$$
\mu_{*}:=\frac{1}{G_{\infty}}\left(\frac{M_{*}}{2 M^{2}}-\lambda \cdot \liminf _{\xi \rightarrow+\infty} \frac{\int_{0}^{T} \max _{|u| \leq \xi} F(t, u) d t}{\xi^{2}}\right),
$$

with $\mu_{*}=+\infty$ when $G_{\infty}=0$, then the problem (IP) has an unbounded sequence of weak solutions in $X$.

Proof From (H3) and the definitions of $\lambda_{1}, \lambda_{2}$ it follows $\lambda_{1}<\lambda_{2}$, and $\mu^{*}>0$ if $\lambda_{1}<\lambda_{2}$. Now fix $\lambda$ and $\mu$ as in the conclusion, $g$ satisfying our assumptions. Our aim is to apply part (b) of Lemma 2.4 to (IP). To this end, consider the functionals $\Phi, \Psi$ defined in (2.2) and (2.3), respectively.

Let $\left\{u_{n}\right\} \subset X, u_{n} \rightarrow u$ in $X$, then it converges uniformly to $u$ on $[0, T]$ and $\liminf _{n \rightarrow \infty}\left\|u_{n}\right\|_{X} \geq\|u\|_{X}$. Thus

$$
\begin{aligned}
\liminf _{n \rightarrow \infty} \Phi\left(u_{n}\right)= & \liminf _{n \rightarrow \infty}\left(\frac{1}{2}\left\|u_{n}\right\|_{X}^{2}+\sum_{j=1}^{l} \int_{0}^{u_{n}^{\prime}\left(t_{j}\right)} I_{1 j}(s) d s+\sum_{j=1}^{l} \int_{0}^{u_{n}\left(t_{j}\right)} I_{2 j}(s) d s\right. \\
& \left.+\frac{\alpha_{1}}{2 \beta_{1}}\left(u_{n}(0)\right)^{2}+\frac{\alpha_{2}}{2 \beta_{2}}\left(u_{n}(T)\right)^{2}\right) \\
\geq & \left(\frac{1}{2}\|u\|_{X}^{2}+\sum_{j=1}^{l} \int_{0}^{u^{\prime}\left(t_{j}\right)} I_{1 j}(s) d s+\sum_{j=1}^{l} \int_{0}^{u\left(t_{j}\right)} I_{2 j}(s) d s\right.
\end{aligned}
$$




$$
\begin{aligned}
& \left.+\frac{\alpha_{1}}{2 \beta_{1}}(u(0))^{2}+\frac{\alpha_{2}}{2 \beta_{2}}(u(T))^{2}\right) \\
= & \Phi(u) .
\end{aligned}
$$

Therefore $\Phi$ is sequentially weakly lower semicontinuous.

For any $u \in X$, it follows from (H0) that

$$
\left|\sum_{j=1}^{l} \int_{0}^{u^{\prime}\left(t_{j}\right)} I_{1 j}(s) d s\right| \leq \frac{k_{1}}{2} \max _{j \in\{1,2, \ldots, l\}}\left|u^{\prime}\left(t_{j}\right)\right|^{2}
$$

and

$$
\left|\sum_{j=1}^{l} \int_{0}^{u\left(t_{j}\right)} I_{2 j}(s) d s\right| \leq \frac{k_{2}}{2} \max _{j \in\{1,2, \ldots, l\}}\left|u\left(t_{j}\right)\right|^{2}
$$

Thus, by Lemma 2.1 we obtain

$$
\begin{aligned}
\Phi(u) & \geq \frac{1}{2}\|u\|_{X}^{2}-\frac{k_{1}}{2}\left\{\max _{j \in\{1,2, \ldots, l\}}\left|u^{\prime}\left(t_{j}\right)\right|^{2}\right\}-\frac{k_{2}}{2}\left\{\max _{j \in\{1,2, \ldots, l\}}\left|u\left(t_{j}\right)\right|^{2}\right\} \\
& \geq \frac{1}{2}\left[1-\left(k_{1}+k_{2}\right) M^{2}\right]\|u\|_{X}^{2}=\frac{M_{*}}{2}\|u\|_{X}^{2} .
\end{aligned}
$$

So $\Phi$ is coercive.

On the other hand, if $u_{n} \rightarrow u$ in $X$ then compactness of embedding $X \hookrightarrow C([0, T])$ implies $u_{n} \rightarrow u$ in $C([0, T])$ i.e., $u_{n} \rightarrow u$ uniformly on $[0, T]$. Thus there is $r>0$ such that $\left\|u_{n}\right\|_{0} \leq r$ for any $n \in \mathbf{N}$. Since $F(t, u)$ and $G(t, u)$ are differentiable with respect to $u$ for a.e. $t \in[0, T]$. Then $F\left(t, u_{n}(t)\right) \rightarrow F(t, u(t))$ and $G\left(t, u_{n}(t)\right) \rightarrow G(t, u(t))$ a.e. $t \in[0, T]$. It follows from the assumption (A3) on $f$ and $g$ that

$$
F\left(t, u_{n}(t)\right) \leq r l_{r}^{(f)}(t) \text { and } \quad G\left(t, u_{n}(t)\right) \leq r l_{r}^{(g)}(t)
$$

where $l_{r}^{(f)}(t):=\sup _{|u| \leq r}|f(t, u)|$ and $l_{r}^{(g)}(t):=\sup _{|u| \leq r}|g(t, u)|$ belong to $L^{2}(0, T)$. By the Lebesgue convergence theorem

$$
\begin{aligned}
\Psi\left(u_{n}\right) & =\int_{0}^{T} F\left(t, u_{n}(t)\right) d t+\frac{\mu}{\lambda} \int_{0}^{T} G\left(t, u_{n}(t)\right) d t \\
& \rightarrow \int_{0}^{T} F(t, u(t)) d t+\frac{\mu}{\lambda} \int_{0}^{T} G(t, u(t)) d t=\Psi(u),
\end{aligned}
$$

which implies $\Psi$ is a sequentially weakly continuous functional on $X$. Hence the functionals $\Phi$ and $\Psi$ satisfy the regularity assumptions of Lemma 2.4 .

Let $\left\{\xi_{n}\right\}$ be a sequence of positive numbers such that $\lim _{n \rightarrow \infty} \xi_{n}=+\infty$ and

$$
\lim _{n \rightarrow+\infty} \frac{\int_{0}^{T} \max _{|u| \leq \xi_{n}} F(t, u) d t}{\xi_{n}^{2}}=\liminf _{\xi \rightarrow+\infty} \frac{\int_{0}^{T} \max _{|u| \leq \xi} F(t, u) d t}{\xi^{2}} .
$$


Put $r_{n}:=\frac{M_{*}}{2 M^{2}} \xi_{n}^{2}$ for all $n \in \mathbf{N}$. Then, for every $v \in X$ with $\Phi(v)<r_{n}$, according to (3.2), one has

$$
\|v\|_{X} \leq \sqrt{\frac{2 \Phi(v)}{M_{*}}} \leq \sqrt{\frac{2 r_{n}}{M_{*}}}=\frac{\xi_{n}}{M},
$$

which implies

$$
\|v\|_{0} \leq \xi_{n}
$$

Note that $\Phi(0)=\Psi(0)=0$. Thus, for all $n \in \mathbf{N}$

$$
\begin{aligned}
\varphi\left(r_{n}\right) & =\inf _{u \in \Phi^{-1}\left(-\infty, r_{n}\right)} \frac{\left(\sup _{v \in \Phi^{-1}\left(-\infty, r_{n}\right)} \Psi(v)\right)-\Psi(u)}{r_{n}-\Phi(u)} \\
& \leq \frac{\sup _{v \in \Phi^{-1}\left[0, r_{n}\right]} \Psi(v)}{r_{n}} \\
& \leq \frac{\int_{0}^{T} \max _{|x| \leq \xi_{n}} F(t, x) d t+\frac{\mu}{\lambda} \int_{0}^{T} \max _{|x| \leq \xi_{n}} G(t, x) d t}{\frac{M_{*}}{2 M^{2}} \xi_{n}^{2}} \\
& =\frac{2 M^{2}}{M_{*}}\left\{\frac{\int_{0}^{T} \max _{|x| \leq \xi_{n}} F(t, x) d t}{\xi_{n}^{2}}+\frac{\mu}{\lambda} \frac{\int_{0}^{T} \max _{|x| \leq \xi_{n}} G(t, x) d t}{\xi_{n}^{2}}\right\} .
\end{aligned}
$$

Hence

$$
\begin{aligned}
\gamma & \leq \liminf _{n \rightarrow+\infty} \varphi\left(r_{n}\right) \\
& \leq \frac{2 M^{2}}{M_{*}}\left\{\liminf _{\xi \rightarrow+\infty} \frac{\int_{0}^{T} \max _{|x| \leq \xi} F(t, x) d t}{\xi^{2}}+\frac{\mu}{\lambda} G_{\infty}\right\} \\
& <+\infty,
\end{aligned}
$$

when $G_{\infty}>0$. It follows from the assumption $\mu \in\left[0, \mu_{*}\right)$ that

$$
\gamma<\frac{2 M^{2}}{M_{*}}\left\{\liminf _{\xi \rightarrow+\infty} \frac{\int_{0}^{T} \max _{|x| \leq \xi} F(t, x) d t}{\xi^{2}}+\frac{\mu_{*}}{\lambda} G_{\infty}\right\}=\frac{1}{\lambda} .
$$

When $G_{\infty}=0$, by $\lambda \in\left(\lambda_{1}, \lambda_{2}\right)$, one has

$$
\gamma<\frac{2 M^{2}}{M_{*}} \liminf _{\xi \rightarrow+\infty} \frac{\int_{0}^{T} \max _{|x| \leq \xi} F(t, x) d t}{\xi^{2}}<\frac{1}{\lambda} .
$$

Hence, $\lambda \in\left(0, \frac{1}{\gamma}\right)$.

Next we shall show that the functional $E_{\lambda, \mu}$ is unbounded from below. Let $\left\{\eta_{n}\right\}$ be a sequence of positive numbers such that $\eta_{n} \rightarrow+\infty$ as $n \rightarrow+\infty$. For all $n \in \mathbf{N}$ define $v_{n} \in X$ by setting

$$
v_{n}(t):= \begin{cases}\frac{4 \eta_{n}}{\alpha_{1} T+4 \beta_{1}}\left(\beta_{1}+\alpha_{1} t\right), & \text { for } t \in\left[0, \frac{T}{4}\right), \\ \eta_{n}, & \text { for } t \in\left[\frac{T}{4}, \frac{3 T}{4}\right), \\ \frac{4 \eta_{n}}{\alpha_{2} T+4 \beta_{2}}\left(T \alpha_{2}+\beta_{2}-\alpha_{2} t\right), & \text { for } t \in\left[\frac{3 T}{4}, T\right] .\end{cases}
$$


For any fixed $n \in \mathbf{N}$, a direct calculation shows that

$$
\begin{aligned}
\Phi\left(v_{n}\right)= & \frac{1}{2}\left\|v_{n}\right\|_{X}^{2}+\sum_{j=1}^{l} \int_{0}^{v_{n}^{\prime}\left(t_{j}\right)} I_{1 j}(s) d s+\sum_{j=1}^{l} \int_{0}^{v_{n}\left(t_{j}\right)} I_{2 j}(s) d s \\
& +\frac{\alpha_{1}}{2 \beta_{1}}\left(v_{n}(0)\right)^{2}+\frac{\alpha_{2}}{2 \beta_{2}}\left(v_{n}(T)\right)^{2} \\
\leq & \frac{1}{2}\left\|v_{n}\right\|_{X}^{2}+\frac{k_{1}+k_{2}}{2}\left\|v_{n}\right\|_{0}^{2}+\frac{\alpha_{1}}{2 \beta_{1}}\left(\frac{4 \beta_{1}}{\alpha_{1} T+4 \beta_{1}}\right)^{2} \eta_{n}^{2}+\frac{\alpha_{2}}{2 \beta_{2}}\left(\frac{4 \beta_{2}}{\alpha_{2} T+4 \beta_{2}}\right)^{2} \eta_{n}^{2} \\
\leq & \left(\Gamma(T)+\frac{k_{1}+k_{2}}{2} M^{2}+\frac{8 \alpha_{1} \beta_{1}}{\left(4 \beta_{1}+\alpha_{1} T\right)^{2}}+\frac{8 \alpha_{2} \beta_{2}}{\left(4 \beta_{2}+\alpha_{2} T\right)^{2}}\right) \eta_{n}^{2} \\
= & \hbar(T, M) \eta_{n}^{2} .
\end{aligned}
$$

On the other hand, by applying condition (H2), the definition of $G_{\infty}$, and the nonnegativity of $G$, one infers that

$$
\begin{aligned}
\Psi\left(v_{n}\right)= & \int_{0}^{T}\left(F\left(t, v_{n}(t)\right)+\frac{\mu}{\lambda} G\left(t, v_{n}(t)\right)\right) d t \\
\geq & \int_{0}^{T} F\left(t, v_{n}(t)\right) d t \\
= & \int_{0}^{T / 4} F\left(t, \frac{4 \eta_{n}\left(\alpha_{2}+\alpha_{1} t\right)}{\alpha_{1} T+4 \alpha_{2}}\right) d t+\int_{3 T / 4}^{T} F\left(t, \frac{4 \eta_{n}\left(T \beta_{1}+\beta_{2}-\beta_{1} t\right)}{\beta_{1} T+4 \beta_{2}}\right) d t \\
& +\int_{T / 4}^{3 T / 4} F\left(t, \eta_{n}\right) d t \\
\geq & \int_{T / 4}^{3 T / 4} F\left(t, \eta_{n}\right) d t .
\end{aligned}
$$

Therefore,

$$
E_{\lambda, \mu}\left(v_{n}\right)=\Phi\left(v_{n}\right)-\lambda \Psi\left(v_{n}\right) \leq \hbar(T, M) \eta_{n}^{2}-\lambda \int_{T / 4}^{3 T / 4} F\left(t, \eta_{n}\right) d t
$$

At this point, put

$$
Q_{\infty}:=\limsup _{\xi \rightarrow+\infty} \frac{\int_{T / 4}^{3 T / 4} F(t, \xi) d t}{\xi^{2}} .
$$

Then we have the following two cases:

Case 1. If $Q_{\infty}<+\infty$, there exists $\varepsilon$ that satisfies

$$
0<\varepsilon<Q_{\infty}-\frac{\hbar(T, M)}{\lambda} .
$$

From (3.8), there is $N_{1}>0$ such that

$$
0<\left(Q_{\infty}-\varepsilon\right) \eta_{n}^{2}<\int_{T / 4}^{3 T / 4} F\left(t, \eta_{n}\right) d t, \quad \forall n>N_{1}
$$


So

$$
E_{\lambda, \mu}\left(v_{n}\right)<\left[\hbar(T, M)-\lambda\left(Q_{\infty}-\varepsilon\right)\right] \eta_{n}^{2}
$$

for every $n>N_{1}$. According to the choice of $\varepsilon$, one has $\lim _{n \rightarrow+\infty} E_{\lambda, \mu}\left(v_{n}\right)=-\infty$.

Case 2. If $Q_{\infty}=+\infty$, take

$$
\sigma>\frac{\hbar(T, M)}{\lambda}
$$

Then there exists $N(\sigma)>0$ such that

$$
\int_{T / 4}^{3 T / 4} F\left(t, \eta_{n}\right) d t>\sigma \eta_{n}^{2}, \quad \forall n>N(\sigma)
$$

Hence

$$
E_{\lambda, \mu}\left(v_{n}\right)<[\hbar(T, M)-\lambda \sigma] \eta_{n}^{2}
$$

for every $n>N(\sigma)$. By the choice of $\sigma$, one infers that

$$
\lim _{n \rightarrow+\infty} E_{\lambda, \mu}\left(v_{n}\right)=-\infty
$$

Therefore, the functional $E_{\lambda, \mu}$ is unbounded from below. By using the case (b) of Lemma 2.4, the functional $E_{\lambda, \mu}$ has a sequence of critical points such that $\lim _{n \rightarrow+\infty}\left\|v_{n}\right\|_{X}=$ $+\infty$ and the conclusion is obtained.

Theorem 3.2 Letf $:[0, T] \times \mathbf{R} \rightarrow \mathbf{R}$ be an $L^{2}$-Carathéodory function and $(\mathrm{H} 1)$ be satisfied . Furthermore, assume that:

(H4) There exist $a \in L^{2}([0,1]), b, d$, and $\theta \in[0,1)$ such that

$$
f(t, u) \geq a(t)-b|u|^{\theta}, \quad \text { for every }(t, u) \in\left(\left[0, \frac{T}{4}\right] \cup\left[\frac{3 T}{4}, T\right]\right) \times[d,+\infty) ;
$$

(H5)

$$
\liminf _{\xi \rightarrow+\infty} \frac{\left\|l_{\xi}\right\|_{L^{2}}}{\xi}<\frac{M_{*}}{2(1+T) M \hbar(T, M)} \cdot \limsup _{\xi \rightarrow+\infty} \frac{\int_{T / 4}^{3 T / 4} F(t, \xi) d t}{\xi^{2}}
$$

where $l_{\xi}(t)=\sup _{|u| \leq \xi}|f(t, u)|$.

Finally, let $\lambda_{1}$ be as in (3.1). Then $\lambda_{1}<\frac{M_{*}}{2(1+T) M \cdot \liminf f_{\xi \rightarrow+\infty} \frac{\left\|l_{\xi}\right\|_{L} L^{2}}{\xi}}$ and for every

$$
\lambda \in \Lambda^{\prime}:=\left(\lambda_{1}, \frac{M_{*}}{2(1+T) M \cdot \liminf _{\xi \rightarrow+\infty} \frac{\left\|l_{\xi}\right\|_{L^{2}}}{\xi}}\right),
$$

and every $L^{2}$-Carathéodory function $g:[0, T] \times \mathbf{R} \rightarrow \mathbf{R}$ such that

$$
G(t, u) \geq 0 \quad \text { for all }(t, u) \in[0, T] \times \mathbf{R},
$$




$$
G_{\infty}:=\limsup _{\xi \rightarrow+\infty} \frac{\int_{0}^{T} \max _{|u| \leq \xi} G(t, u) d t}{\xi^{2}}<+\infty
$$

and every $\mu \in\left[0, \mu_{*}\right)$, where

$$
\mu_{*}:=\frac{1}{G_{\infty}}\left(\frac{M_{*}}{2 M^{2}}-\frac{\lambda(1+T)}{M} \cdot \liminf _{\xi \rightarrow+\infty} \frac{\left\|l_{\xi}\right\|_{L^{2}}}{\xi}\right),
$$

with $\mu_{*}=+\infty$ when $G_{\infty}=0$, then the problem (IP) has an unbounded sequence of weak solutions in $X$.

Proof Assume that $\left\{\xi_{n}\right\}$ is a sequence of positive numbers such that $\lim _{n \rightarrow \infty} \xi_{n}=+\infty$ and

$$
\lim _{n \rightarrow+\infty} \frac{\left\|l_{\xi_{n}}\right\|_{L^{2}}}{\xi_{n}}=\liminf _{\xi \rightarrow+\infty} \frac{\left\|l_{\xi}\right\|_{L^{2}}}{\xi} .
$$

It follows from (3.3) and condition (A3) that

$$
|F(t, u)| \leq\left|\int_{0}^{u(t)} l_{\xi_{n}}(s) d s\right|=|u(t)| \cdot\left|l_{\xi_{n}}(t)\right| .
$$

By the Hölder inequality,

$$
\begin{aligned}
\left|\int_{0}^{T} F(t, u(t)) d t\right| & \leq \int_{0}^{T}|u(t)| \cdot\left|l_{\xi_{n}}(t)\right| d t \\
& \leq\left(\int_{0}^{T}|u(t)|^{2} d t\right)^{1 / 2} \cdot\left(\int_{0}^{T}\left|l_{\xi_{n}}(t)\right|^{2} d t\right)^{1 / 2} \\
& \leq(1+T)\|u\|_{X} \cdot\left\|l_{\xi_{n}}\right\|_{L^{2}} .
\end{aligned}
$$

By the same argument as given in Theorem 3.1, one has

$$
\begin{aligned}
\gamma & \leq \liminf _{n \rightarrow+\infty}\left\{\inf _{u \in \Phi^{-1}\left(-\infty, r_{n}\right)} \frac{\left(\sup _{v \in \Phi^{-1}\left(-\infty, r_{n}\right)} \Psi(v)\right)-\Psi(u)}{r_{n}-\Phi(u)}\right\} \\
& \leq \liminf _{n \rightarrow+\infty}\left\{\frac{\sup _{v \in \Phi^{-1}\left[0, r_{n}\right]} \Psi(v)-\Psi(u)}{r_{n}-\Phi(u)}\right\} \\
& \leq \frac{2(1+T) M}{M_{*}} \liminf _{n \rightarrow+\infty} \frac{\left\|l_{\xi n}\right\|_{L^{2}}}{\xi_{n}}+\frac{2 M^{2} \mu}{\lambda M_{*}} \limsup _{n \rightarrow+\infty} \frac{\int_{0}^{T} \max _{|x| \leq \xi_{n}} G(t, x) d t}{\xi_{n}^{2}} \\
& =\frac{2(1+T) M}{M_{*}} \liminf _{\xi \rightarrow+\infty} \frac{\left\|l_{\xi}\right\|_{L^{2}}}{\xi}+\frac{2 M^{2} \mu}{\lambda M_{*}} G_{\infty} \\
& \leq \frac{2(1+T) M}{M_{*}} \liminf _{\xi \rightarrow+\infty} \frac{\left\|l_{\xi}\right\|_{L^{2}}}{\xi}+\frac{2 M^{2} \mu_{*}}{\lambda M_{*}} G_{\infty}=\frac{1}{\lambda},
\end{aligned}
$$

when $G_{\infty}>0$ and $\mu \in\left[0, \mu_{*}\right)$. When $G_{\infty}=0$, it follows from $\lambda \in \Lambda^{\prime}$ that

$$
\gamma<\frac{2(1+T) M}{M_{*}} \liminf _{\xi \rightarrow+\infty} \frac{\left\|l_{\xi}\right\|_{L^{2}}}{\xi}<\frac{1}{\lambda} .
$$

Therefore, $\lambda \in\left(0, \frac{1}{\gamma}\right)$. 
On the other hand, if $\lambda \in \Lambda^{\prime}$, then there are a constant $\kappa$ and a sequence of positive numbers $\left\{\eta_{n}\right\}$ with $\lim _{n \rightarrow \infty} \eta_{n}=+\infty$, such that

$$
\hbar(T, M)<\kappa<\lambda \lim _{n \rightarrow+\infty} \frac{\int_{T / 4}^{3 T / 4} F\left(t, \eta_{n}\right) d t}{\eta_{n}^{2}}=\lambda \lim \sup _{\xi \rightarrow+\infty} \frac{\int_{T / 4}^{3 T / 4} F(t, \xi) d t}{\xi^{2}} .
$$

According to assumption (H4), we have

$$
F(t, u(t)) \geq-|a(t)| \cdot|u(t)|-\frac{b}{1+\theta} \cdot|u(t)|^{1+\theta}-|u(t)| \cdot\left|l_{d}(t)\right|, \quad \forall t \in\left[0, \frac{T}{4}\right) \cup\left(\frac{3 T}{4}, T\right] .
$$

From the Hölder inequality and Lemma 2.1, we get

$$
\begin{aligned}
& \int_{0}^{T / 4} F(t, u(t)) d t+\int_{0}^{3 T / 4} F(t, u(t)) d t \\
& \quad \geq-(1+T)\left(\|a\|_{L^{2}}+\left\|l_{d}\right\|_{L^{2}}\right)\|u\|_{X}-\frac{b(1+T)^{1+\theta} \sqrt{T}\|u\|_{X}^{1+\theta}}{1+\theta} .
\end{aligned}
$$

It follows from (3.5), (3.10), and the nonnegativity of $G$ that

$$
\begin{aligned}
E_{\lambda, \mu}\left(v_{n}\right) \leq & \hbar(T, M) \eta_{n}^{2}-\lambda \int_{0}^{T} F(t, u(t)) d t \\
\leq & \hbar(T, M) \eta_{n}^{2}-\lambda \int_{T / 4}^{3 T / 4} F(t, u(t)) d t \\
& +\lambda\left\{(1+T)\left(\|a\|_{L^{2}}+\left\|l_{d}\right\|_{L^{2}}\right)\|u\|_{X}+\frac{(1+T)^{1+\theta} \sqrt{T} b}{1+\theta}\|u\|_{X}^{1+\theta}\right\} \\
\leq & (\hbar(T, M)-\kappa) \eta_{n}^{2} \\
& +\lambda\left\{(1+T)\left(\|a\|_{L^{2}}+\left\|l_{d}\right\|_{L^{2}}\right)\|u\|_{X}+\frac{(1+T)^{1+\theta} \sqrt{T} b}{1+\theta}\|u\|_{X}^{1+\theta}\right\} .
\end{aligned}
$$

Since $\theta \in[0,1)$ and $\eta_{n} \rightarrow \infty$ as $n \rightarrow \infty$, (3.9) implies that the functional $E_{\lambda, \mu}$ is unbounded from below and the conclusion is obtained.

The following results are three special cases of Theorem 3.1.

Corollary 3.3 Let $f:[0, T] \times \mathbf{R} \rightarrow \mathbf{R}$ be an $L^{2}$-Carathéodory function. Assume that hypotheses (H1), (H2) in Theorem 3.1 hold and

$$
\begin{aligned}
& \liminf _{\xi \rightarrow+\infty} \frac{\int_{0}^{T} \max _{|u| \leq \xi} F(t, u) d t}{\xi^{2}}<\frac{M_{*}}{2 M^{2}}, \\
& \limsup _{\xi \rightarrow+\infty} \frac{\int_{T / 4}^{3 T / 4} F(t, \xi) d t}{\xi^{2}}>\hbar(T, M) .
\end{aligned}
$$

For every arbitrary $L^{2}$-Carathéodory function $g:[0, T] \times \mathbf{R} \rightarrow \mathbf{R}$ such that

$$
G(t, u) \geq 0 \quad \text { for all }(t, u) \in[0, T] \times \mathbf{R},
$$




$$
G_{\infty}:=\limsup _{\xi \rightarrow+\infty} \frac{\int_{0}^{T} \max _{|u| \leq \xi} G(t, u) d t}{\xi^{2}}<+\infty,
$$

and every $\mu \in\left[0, \mu_{*}\right)$, where

$$
\mu_{*}:=\frac{1}{G_{\infty}}\left(\frac{M_{*}}{2 M^{2}}-\liminf _{\xi \rightarrow+\infty} \frac{\int_{0}^{T} \max _{|u| \leq \xi} F(t, u) d t}{\xi^{2}}\right),
$$

with $\mu_{*}=+\infty$ when $G_{\infty}=0$, the equation

$$
u^{(i v)}(t)-u^{\prime \prime}(t)+u(t)=f(t, u(t))+\mu g(t, u(t)), \quad t \neq t_{j}, \text { a.e. } t \in[0, T]
$$

with boundary conditions (1.2) and impulsive conditions (1.3) admits an unbounded sequence of weak solutions in $X$.

Corollary 3.4 Let $f:[0, T] \times \mathbf{R} \rightarrow \mathbf{R}$ be an $L^{2}$-Carathéodory function. Assume that hypotheses (H1), (H2) in Theorem 3.1 hold and

$$
\liminf _{\xi \rightarrow+\infty} \frac{\int_{0}^{T} \max _{|u| \leq \xi} F(t, u) d t}{\xi^{2}}=0 \text { and } \limsup _{\xi \rightarrow+\infty} \frac{\int_{T / 4}^{3 T / 4} F(t, \xi) d t}{\xi^{2}}=+\infty .
$$

Then for every nonnegative continuous function $g: \mathbf{R} \rightarrow \mathbf{R}$ satisfying the condition

$$
G_{\infty}:=\lim _{\xi \rightarrow+\infty} \frac{\int_{0}^{\xi} g(x) d x}{\xi^{2}}<+\infty
$$

and for every $\mu \in\left[0, \frac{M_{*}}{2 M^{2} G_{\infty}}\right)$, the equation

$$
u^{(i v)}(t)-u^{\prime \prime}(t)+u(t)=f(t, u(t))+\mu g(u(t)), \quad t \neq t_{j}, \text { a.e. } t \in[0, T],
$$

with boundary conditions (1.2) and impulsive conditions (1.3) has an unbounded sequence of weak solutions in $X$.

Let $q: \mathbf{R} \rightarrow \mathbf{R}$ be a nonnegative continuous function and $p \in L^{2}([0, T])$ such that $p(t) \geq 0$ a.e. $t \in[0, T]$ and $p \not \equiv 0$. Furthermore, put

$$
Q(u):=\int_{0}^{u} q(x) d x, \quad \text { for all } u \in \mathbf{R}, \quad p_{0}:=\int_{T / 4}^{3 T / 4} p(t) d t .
$$

Then we have the following special situation of Theorem 3.1 when $\mu=0$.

Corollary 3.5 Let $f:[0, T] \times \mathbf{R} \rightarrow \mathbf{R}$ be an $L^{2}$-Carathéodory function. Assume that hypothesis (H1) in Theorem 3.1 holds and

$$
\liminf _{\xi \rightarrow+\infty} \frac{Q(\xi)}{\xi^{2}} \leq \frac{p_{0} M_{*}}{2 \sqrt{T} M^{2}\|p\|_{L^{2}} \hbar(T, M)} \cdot \limsup _{\xi \rightarrow+\infty} \frac{Q(\xi)}{\xi^{2}} .
$$

Then for every

$$
\lambda \in\left(\frac{\hbar(T, M)}{p_{0} \lim \sup _{\xi \rightarrow+\infty} \frac{Q(\xi)}{\xi^{2}}}, \frac{M_{*}}{2 \sqrt{T} M^{2}\|p\|_{L^{2}} \liminf _{\xi \rightarrow+\infty} \frac{Q(\xi)}{\xi^{2}}}\right),
$$


the equation

$$
u^{(i v)}(t)-u^{\prime \prime}(t)+u(t)=\lambda p(t) q(u(t)), \quad t \neq t_{j}, \text { a.e. } t \in[0, T],
$$

with boundary conditions (1.2) and impulsive conditions (1.3) has an unbounded sequence of weak solutions in $X$.

In the following, using case (c) of Lemma 2.4 and arguing as in the proof of Theorem 3.1, we can obtain the existence of infinitely many solutions of (IP).

For convenience, set

$$
\begin{aligned}
& \lambda_{3}:= \begin{cases}\frac{\hbar(T, M)}{\limsup _{\xi \rightarrow 0^{+}} \frac{\int_{T / 4}^{3 T / 4} F(t, \xi) d t}{\xi^{2}},}, & \text { if } \limsup _{\xi \rightarrow 0^{+}} \frac{\int_{T T 4}^{3 T / 4} F(t, \xi) d t}{\xi^{2}}<+\infty, \\
0, & \text { if } \limsup _{\xi \rightarrow 0^{+}} \frac{\int_{T / 4}^{3 T / 4} F(t, \xi) d t}{\xi^{2}}=+\infty,\end{cases} \\
& \lambda_{4}:=\frac{M_{*}}{2 M^{2} \cdot \liminf _{\xi \rightarrow 0^{+}} \frac{\int_{0}^{T} \max _{|x| \leq \xi} F(t, x) d t}{\xi^{2}} .}
\end{aligned}
$$

Theorem 3.6 Letf $:[0, T] \times \mathbf{R} \rightarrow \mathbf{R}$ be an $L^{2}$-Carathéodory function and (H1), (H2) hold. Furthermore, assume that

(H6)

$$
\liminf _{\xi \rightarrow 0^{+}} \frac{\int_{0}^{T} \max _{|u| \leq \xi} F(t, u) d t}{\xi^{2}}<\frac{M_{*}}{2 M^{2} \hbar(T, M)} \cdot \limsup _{\xi \rightarrow 0^{+}} \frac{\int_{T / 4}^{3 T / 4} F(t, \xi) d t}{\xi^{2}} .
$$

Then $\lambda_{3}<\lambda_{4}$, and for every arbitrary $\lambda \in \Lambda^{\prime}:=\left(\lambda_{3}, \lambda_{4}\right)$, and for every arbitrary $L^{2}$ Carathéodory function $g:[0, T] \times \mathbf{R} \rightarrow \mathbf{R}$ such that

$$
\begin{aligned}
& G(t, u) \geq 0 \text { for all }(t, u) \in[0, T] \times \mathbf{R}, \\
& G_{0}:=\limsup _{\xi \rightarrow 0^{+}} \frac{\int_{0}^{T} \max _{|u| \leq \xi} G(t, u) d t}{\xi^{2}}<+\infty,
\end{aligned}
$$

and for every $\mu \in\left[0, \mu_{*}^{\prime}\right)$, where

$$
\mu_{*}^{\prime}:=\frac{1}{G_{0}}\left(\frac{M_{*}}{2 M^{2}}-\lambda \cdot \liminf _{\xi \rightarrow 0^{+}} \frac{\int_{0}^{T} \max _{|u| \leq \xi} F(t, u) d t}{\xi^{2}}\right),
$$

with $\mu_{*}^{\prime}=+\infty$ when $G_{0}=0$, the problem $(I P)$ has a sequence $\left\{u_{n}\right\}$ of weak solutions such that $u_{n} \rightarrow 0$.

Proof From (H6) and the definitions of $\lambda_{3}, \lambda_{4}$ it follows $\lambda_{3}<\lambda_{4}$, and $\mu_{*}^{\prime}>0$ if $\lambda_{3}<\lambda_{4}$. Now fix $\lambda$ and $\mu_{*}^{\prime}$ as in the conclusion, $g$ satisfying our assumptions. Our goal is to apply part (c) of Lemma 2.4 to $\Phi$ and $\Psi$ defined in (2.2) and (2.3), respectively. As has been pointed out before, the functionals $\Phi, \Psi$ satisfy the assumptions of regularity required in Lemma 2.4.

Let $\left\{\zeta_{n}\right\}$ be a sequence of positive numbers such that $\lim _{n \rightarrow \infty} \zeta_{n}=0$ and

$$
\lim _{n \rightarrow+\infty} \frac{\int_{0}^{T} \max _{|u| \leq \zeta n} F(t, u) d t}{\zeta_{n}^{2}}=\liminf _{\zeta \rightarrow 0^{+}} \frac{\int_{0}^{T} \max _{|u| \leq \zeta} F(t, u) d t}{\zeta^{2}} .
$$


According to (3.1), we get $\inf _{X} \Phi=0$. By the definition of $\delta$, one has

$$
\delta=\liminf _{r \rightarrow 0^{+}} \varphi(r)
$$

Put $r_{n}:=\frac{M_{*}}{2 M^{2}} \zeta_{n}^{2}$ for all $n \in \mathbf{N}$. Then, for every $v \in X$ with $\Phi(v)<r_{n}$, in view of (3.1), one has $\|v\|_{0} \leq \zeta_{n}$. Moreover, working as in the proof of Theorem 3.1, it follows that $\delta<+\infty$, and also $\Lambda^{\prime} \subset\left(0, \frac{1}{\delta}\right)$.

Now it remains to show that the functional $E_{\lambda, \mu}$ does not have a local minimum at zero.

Let $\left\{\eta_{n}\right\}_{n=1}^{\infty}$ be a sequence in $(0, \tau)(\tau>0)$ such that $\eta_{n} \rightarrow 0$ as $n \rightarrow+\infty$. For all $n \in \mathbf{N}$ define $\left\{\omega_{n}\right\} \in X$ as follows:

$$
\omega_{n}(t):= \begin{cases}\frac{4 \eta_{n}}{\alpha_{1} T+4 \beta_{1}}\left(\beta_{1}+\alpha_{1} t\right), & \text { for } t \in\left[0, \frac{T}{4}\right), \\ \eta_{n}, & \text { for } t \in\left[\frac{T}{4}, \frac{3 T}{4}\right), \\ \frac{4 \eta_{n}}{\alpha_{2} T+4 \beta_{2}}\left(T \alpha_{2}+\beta_{2}-\alpha_{2} t\right), & \text { for } t \in\left[\frac{3 T}{4}, T\right] .\end{cases}
$$

For any fixed $n \in \mathbf{N}$, it follows from condition (H2), the definition of $G_{0}$, and the nonnegativity of $G$ that (3.4) holds.

Put

$$
Q_{0}:=\limsup _{\xi \rightarrow 0^{+}} \frac{\int_{T / 4}^{3 T / 4} F(t, \xi) d t}{\xi^{2}} .
$$

Suppose that $Q_{0}<+\infty$. Then we can choose $\varepsilon$ such that

$$
0<\varepsilon<Q_{0}-\frac{\hbar(T, M)}{\lambda}
$$

From (3.12), there is $N_{2}>0$ such that

$$
0<\left(Q_{0}-\varepsilon\right) \eta_{n}^{2}<\int_{T / 4}^{3 T / 4} F\left(t, \eta_{n}\right) d t, \quad \forall n>N_{2}
$$

So

$$
E_{\lambda, \mu}\left(\omega_{n}\right)<\left[\hbar(T, M)-\lambda\left(Q_{0}-\varepsilon\right)\right] \eta_{n}^{2}
$$

for every $n>N_{2}$. According to the choice of $\varepsilon$, one has $\lim _{n \rightarrow+\infty} E_{\lambda, \mu}\left(\omega_{n}\right)=E_{\lambda, \mu}(0)<0$.

Similarly, for case $Q_{0}=+\infty$, one also has $\lim _{n \rightarrow+\infty} E_{\lambda, \mu}\left(\omega_{n}\right)<0$. Therefore, we find that zero is not a local minimum of the functional $E_{\lambda, \mu}$. Since $\Phi(0)=\inf _{X} \Phi=0$, we deduce that the energy functional $E_{\lambda, \mu}$ does not have a local minimum at the unique global minimum of $\Phi$. So, by Lemma 2.4(c), there is a sequence $\left\{u_{n}\right\}$ of critical points of the functional $E_{\lambda, \mu}$, which converges weakly to zero. Because $X$ is compactly embedded in $C^{1}([0, T])$, it turns out that the critical points converge weakly to zero, and the conclusion is obtained.

Finally, we present an example to illustrate our main results. 
Example 3.7 Consider the following boundary value problem with impulsive effect:

$$
\left\{\begin{array}{l}
u^{(i v)}(t)-u^{\prime \prime}(t)+u(t)=\lambda f(t, u(t))+\mu g(t, u(t)), \quad \text { a.e. } t \in[0,1] \\
\Delta u^{\prime \prime}\left(t_{1}\right)=\frac{1}{320} u^{\prime}\left(t_{1}\right) \cos \left(u^{\prime}\left(t_{1}\right)\right)^{2}, \quad-\triangle u^{\prime \prime \prime}\left(t_{1}\right)=\frac{1}{160} u\left(t_{1}\right), \quad t_{1}=\frac{1}{2} \\
2 u(0)-\frac{3}{2} u^{\prime}(0)=0, \quad \frac{1}{2} u(1)+u^{\prime}(1)=0 \\
2 u^{\prime \prime}(0)-\frac{3}{2} u^{\prime \prime \prime}(0)=0, \quad \frac{1}{2} u^{\prime \prime}(1)+u^{\prime \prime \prime}(1)=0
\end{array}\right.
$$

where $T=1, \alpha_{1}=2, \alpha_{2}=\frac{1}{2}, \beta_{1}=\frac{3}{2}, \beta_{2}=1 ; I_{11}(x)=\frac{1}{320} x \cos x^{2}, I_{21}(x)=\frac{1}{160} x ; f:[0, T] \times \mathbf{R} \rightarrow$ $\mathbf{R}$ and $g:[0, T] \times \mathbf{R} \rightarrow \mathbf{R}$ are $L^{2}$-Carathéodory functions defined as follows:

$$
f(t, x)= \begin{cases}0, & (t, x) \in[0,1] \times\{0\}, \\ t x(37-36 \sin (\ln |x|)-18 \cos (\ln |x|)), & (t, x) \in[0,1] \times\{\mathbf{R}-\{0\}\},\end{cases}
$$

and

$$
g(t, x)= \begin{cases}\frac{2}{9} x^{\frac{1}{5}}+\frac{3}{2} t^{2} x^{\frac{1}{3}}, & (t, x) \in[0,1] \times(-\infty, 1], \\ \frac{3}{2} t^{2} x^{\frac{1}{2}}+\frac{x}{10}\left(\frac{11}{9}+\cos (\ln |x|)-\frac{1}{20} \sin (\ln |x|)\right), & (t, x) \in[0,1] \times(1,+\infty) .\end{cases}
$$

Then, for every $\lambda \in(0.1816,0.4812)$ and $\mu \in[0,1.0829-2.25 \lambda)$, BVP (3.13) has a sequence of weak solutions which is unbounded in

$$
Y=\left\{u \in H^{2}(0,1) \mid 2 u(0)-\frac{3}{2} u^{\prime}(0)=0, \frac{1}{2} u(1)+u^{\prime}(1)=0\right\} .
$$

In fact, let $k_{1}=\frac{1}{320}, k_{2}=\frac{1}{160}$, then condition $(\mathrm{H} 0)$ holds. Moreover, one has

$$
F(t, x)= \begin{cases}0, & (t, x) \in[0,1] \times\{0\}, \\ t x^{2}\left(\frac{37}{2}-18 \sin (\ln |x|)\right), & (t, x) \in[0,1] \times\{\mathbf{R}-\{0\}\},\end{cases}
$$

and

$$
G(t, x)= \begin{cases}\frac{5}{27} x^{\frac{6}{5}}+\frac{9}{8} t^{2} x^{\frac{4}{3}}, & (t, x) \in[0,1] \times(-\infty, 1], \\ \frac{2}{27}+\frac{t^{2}}{8}+t^{2} x^{\frac{4}{3}}+\frac{x^{2}}{20}\left(\frac{11}{9}+\cos (\ln |x|)\right), & (t, x) \in[0,1] \times(1,+\infty) .\end{cases}
$$

It is easy to show that

$$
\begin{aligned}
& \liminf _{\xi \rightarrow+\infty} \frac{\int_{0}^{1} \max _{|u| \leq \xi} F(t, u) d t}{\xi^{2}}=0.25, \quad \limsup _{\xi \rightarrow+\infty} \frac{\int_{1 / 4}^{3 / 4} F(t, \xi) d t}{\xi^{2}}=9.125, \\
& G_{\infty}=\limsup _{\xi \rightarrow+\infty} \frac{\int_{0}^{1} \max _{|u| \leq \xi} G(t, u) d t}{\xi^{2}}=\frac{1}{9} .
\end{aligned}
$$

It is easy to calculate that $M=2, M_{*} \approx 0.9626, \Gamma(T) \approx 1.0660, \hbar(T, M) \approx 1.6573$, and

$$
\begin{gathered}
\lambda_{1}=\frac{\hbar(T, M)}{\lim \sup _{\xi \rightarrow+\infty} \frac{\int_{1 / 4}^{3 / 4} F(t, \xi) d t}{\xi^{2}}} \approx 0.1816 \\
<\lambda_{2}=\frac{M_{*}}{2 M^{2} \cdot \liminf _{\xi \rightarrow+\infty} \frac{\int_{0}^{1} \max _{|x| \leq \xi} F(t, x) d t}{\xi^{2}}} \approx 0.4812,
\end{gathered}
$$


which implies that condition (H3) holds. Obviously, conditions (H1) and (H2) are satisfied. Thus, by Theorem 3.1, for every $\lambda \in(0.1816,0.9626)$ and $\mu \in[0,1.0829-2.25 \lambda)$, the problem (3.13) has an unbounded sequence of solutions in $Y$.

\section{Competing interests}

The authors declare that they have no competing interests.

\section{Authors' contributions}

The authors declare that the study was realized in collaboration with the same responsibility. All authors read and approved the final manuscript.

\section{Acknowledgements}

The authors are highly grateful for the referees' careful reading and comments on this paper. This work was supported by Hunan Provincial Natural Science Foundation of China (No. 13JJ3106), National Natural Science Foundation of China (No. 11201138) and Scientific Research Fund of Hunan Provincial Education Department (No. 12C0088); and it was also supported by Aid program for Science and Technology Innovative Research Team in Higher Educational Institutions of Hunan Province.

\section{Received: 31 January 2015 Accepted: 18 August 2015 Published online: 29 August 2015}

\section{References}

1. Ge, W, Ren, J: New existence theorems of positive solutions for Sturm-Liouville boundary value problems. Appl. Math. Comput. 148, 631-644 (2004)

2. Li, Y: On the existence and nonexistence of positive solutions for nonlinear Sturm-Liouville boundary value problems. J. Math. Anal. Appl. 304, 74-86 (2005)

3. Sun, B, Ge, W: Existence and iteration of positive solutions to a class of Sturm-Liouville-like $p$-Laplacian boundary value problems. Nonlinear Anal. TMA 69, 1454-1461 (2008)

4. Zhao, Y, Chen, H: Multiplicity of solutions to two-point boundary value problems for second-order impulsive differential equation. Appl. Math. Comput. 206, 925-931 (2008)

5. Yang, J, Wei, Z, Liu, K: Existence of symmetric positive solutions for a class of Sturm-Liouville-like boundary value problems. Appl. Math. Comput. 214, 424-432 (2009)

6. Averna, D, Giovannelli, N, Tornatore, E: Existence of three solutions for a mixed boundary value problem with the Sturm-Liouville equation. Bull. Korean Math. Soc. 49, 1213-1222 (2012)

7. Zhang, Q, Li, F, Zhu, X: Multiple sign-changing solutions to the Sturm-Liouville boundary value problem with resonance. Appl. Math. Comput. 219, 1061-1072 (2012)

8. Bonanno, G, Riccobono, G: Multiplicity results for Sturm-Liouville boundary value problems. Appl. Math. Comput. 210, 294-297 (2009)

9. Bonanno, G, Heidarkhani, S, O'Regan, D: Nontrivial solutions for Sturm-Liouville systems via a local minimum theorem for functionals. Bull. Aust. Math. Soc. 89, 8-18 (2014)

10. Graef, JR, Heidarkhani, S, Kong, L: Infinitely many solutions for systems of Sturm-Liouville boundary value problems. Results Math. 66, 327-341 (2014)

11. Nieto, JJ, O'Regan, D: Variational approach to impulsive differential equations. Nonlinear Anal., Real World Appl. 10, 680-690 (2009)

12. Sun, J, Chen, H, Nieto, JJ, Otero-Novoa, M: Multiplicity of solutions for perturbed second-order Hamiltonian systems with impulsive effects. Nonlinear Anal. TMA 72, 4575-4586 (2010)

13. Sun, J, Chen, H, Yang, L: Variational methods to fourth-order impulsive differential equations. J. Appl. Math. Comput. 35, 323-340 (2011)

14. Radulescu, V: Finitely many solutions for a class of boundary value problems with superlinear convex nonlinearity. Arch. Math. (Basel) 84, 538-550 (2005)

15. Zhao, Y, Wang, $X$, Liu, X: New results for perturbed second-order impulsive differential equation on the half-line Bound. Value Probl. 2014, 246 (2014)

16. Molica Bisci, G: Variational problems on the sphere. In: Recent Trends in Nonlinear Partial Differential Equations. Dedicated to Patrizia Pucci on the occasion of her 60th birthday. Contemporary Mathematics, vol. 595, pp. 273-291 (2013)

17. Cabada, A, Tersian, S: Existence and multiplicity of solutions to boundary value problems for fourth-order impulsive differential equations. Bound. Value Probl. 2014, 105 (2014)

18. Ferrara, M, Khademloob, S, Heidarkhani, S: Multiplicity results for perturbed fourth-order Kirchhoff type elliptic problems. Appl. Math. Comput. 234, 316-325 (2014)

19. Tian, Y, Ge, W: Variational methods to Sturm-Liouville boundary value problem for impulsive differential equations. Nonlinear Anal. TMA 72, 277-287 (2009)

20. Yan, L, Luo, Z, Liu, J: Multiplicity of solutions for second-order impulsive differential equations with Sturm-Liouville boundary conditions. Adv. Differ. Equ. 2014, 49 (2014)

21. Afrouzi, GA, Hadjian, A, Rădulescu, V: Variational approach to fourth-order impulsive differential equations with two control parameters. Results Math. 65, 371-384 (2014)

22. Tian, Y, Ge, W: Multiple solutions of impulsive Sturm-Liouville boundary value problem via lower and upper solutions and variational methods. J. Math. Anal. Appl. 387, 475-489 (2012)

23. Tian, Y, Liu, X: Applications of variational methods to Sturm-Liouville boundary-value problem for fourth-order impulsive differential equations. Math. Methods Appl. Sci. 37, 95-105 (2014)

24. Bonanno, G, Molica Bisci, G: Infinitely many solutions for a boundary value problems with discontinuous nonlinearities. Bound. Value Probl. 2009670675 (2009)

25. Ricceri, B: A general variational principle and some of its applications. J. Comput. Appl. Math. 113, $401-410$ (2000) 